\title{
Analysis of joint channel estimation and joint data detection in TD-SCDMA systems
}

\begin{abstract}
Time Division-Synchronous Code Division Multiple Access (TD-SCDMA) has several attractive characteristics that made it capable of meeting the requirements of G4 systems when applied as a platform directly. Joint detections, the unpaired spectrums with flexible sharing of resources, and some other advanced properties leads TD-SCDMA to achieve high data rate services. In this paper a comprehensive analysis is presented that involve accurate Joint Channel Estimation (JCE) for traditional and enhanced estimators based on two criterions: Least square (LS) \& Minimum Mean Square Error (MMSE) at two scenarios of users' movements. In addition to a comprehensive Multi-Users Joint Detection (MUJD) is improved to mitigate Multiple Access Interference (MAI) and Inter-Symbol Interference (ISI) using the following detectors: Matched Filter (MF), Zero Forcing Block Linear Equalizer (ZF-BLE), and Minimum Mean Square Error Block Linear Equalizer (MMSE-BLE) for traditional and enhanced channel estimation criterions. Simulation results showed that equalizers fed by Channel Impulse Response (CIR) optimized according to enhanced LS or MMSE give a better performance to that ones when CIR obtained from traditional LS or MMSE criterion. The using of MMSE give the opportunity to implement adaptive equalizes to cope with fast fading and/or non stationary interference environments.
\end{abstract}

Keyword: TD-SDMA system; Joint channel estimation (JCE); Multi-users joint detection (MUJD) 